\title{
Proceedings
}

\section{Testing the Reliability of the Soft-Gluon Approximation for High $p_{\perp}$ Particles ${ }^{\dagger}$}

\author{
Bojana Blagojevic $^{1} *\left(\mathbb{D}\right.$, Magdalena Djordjevic ${ }^{1}$ and Marko Djordjevic ${ }^{2}$ \\ 1 Institute of Physics Belgrade, University of Belgrade, 11000 Belgrade, Serbia; magda@ipb.ac.rs \\ 2 Faculty of Biology, Institute of Physiology and Biochemistry, University of Belgrade, 11000 Belgrade, Serbia; \\ dmarko@bio.bg.ac.rs \\ * Correspondence: bojanab@ipb.ac.rs; Tel.: +381-11-37-13-086 \\ + Presented at Hot Quarks 2018-Workshop for Young Scientists on the Physics of Ultrarelativistic \\ Nucleus-Nucleus Collisions, Texel, The Netherlands, 7-14 September 2018.
}

Published: 11 April 2019

\begin{abstract}
The soft-gluon approximation assumes that a high $p_{\perp}$ parton propagating through dense QCD matter loses only a small amount of its energy via gluon radiation. This assumption is made in many different jet quenching approaches, which nevertheless predicted a sizable radiative energy loss of such particles. This questions the reliability of this approximation, which must then be reconsidered. To address this issue, we relaxed the soft-gluon approximation within the DGLV formalism to the first order in opacity. The obtained analytical expressions are notably different from the soft-gluon case. Surprisingly the numerical effects that stem from waiving this assumption on fractional radiative energy loss and number of radiated gluons are small. Additionally, the effect on suppression is negligible, which can be intuitively understood by the cancellation of the opposite effects on the above mentioned variables. Consequently, our results surprisingly indicate that, contrary to the doubts mentioned above, the soft-gluon approximation remains well-founded within the DGLV formalism. We also investigate the effects of this assumption in the case of a dynamical medium, which suggests generality of the conclusions presented here.
\end{abstract}

Keywords: beyond soft-gluon approximation; radiative energy loss; high $p_{\perp}$ particles; suppression

\section{Introduction}

The soft-gluon $(\mathrm{sg})$ approximation, which assumes that radiated gluon $(\omega)$ takes away only small fraction $\left(x=\frac{\omega}{E} \ll 1\right)$ of an initial parton's energy $(E)$, was commonly used in various energy loss models [1-4]. These models obtained a significant radiative energy loss, indicating possible inconsistency with the applied approximation. To this end, some of the models ("effectively") relaxed the approximation [5-8], which lead to non-unified conclusion on the reliability of this approximation. On the other hand, our dynamical energy loss model [9], enclosing the same approximation, reported a robust agreement with comprehensive set of experimental suppression data [9,10], implicitly suggesting applicability of such approximation. However, the approximation clearly breaks-down for intermediate momentum range $\left(5<p_{\perp}<10 \mathrm{GeV}\right)$, where the experimental data are most abundant and with the smallest error bars; and primarily for the gluon-jets, as due to the color factor of $9 / 4$, gluons lose significantly more energy compared to the quarks. This questioned the applicability of the soft-gluon approximation (sga), which we addressed in [11] for the gluon-jet radiative energy loss within the DGLV [12] formalism to the 1st order in opacity, by allowing $x$ to acquire a finite value, and discussed its implications when recoiling of the medium constituents is taken into account. Note that, in these proceedings, only the main results are presented, while for more details, we refer the reader to [11]. 


\section{Methods}

In this section we outline the features of the DGLV formalism, the assumptions used in the beyond soft-gluon (bsg) calculations [11], as well as the definition of an observable for which we generated the predictions. The DGLV formalism [12] considers a finite size, optically thin QCD medium, consisting of static scattering centers, so that the jet-medium interactions are described by static color-screened Yukawa potential given by Eq. (5) from [13], whereas gluons in a finite temperature QGP are considered to be transversely polarized with an effective mass equal to $m_{g}=\mu / \sqrt{2}$ ( $\mu$ is Debye mass), as obtained in [14]. We applied the following assumptions in calculating 11 Feynman diagrams (for more details see [11]) for the gluon-jet $b s g$ approximation: (1) that initial gluon propagates along the longitudinal axis consistently for all diagrams; (2) the soft-rescattering (eikonal) approximation and (3) the first order in opacity approximation.

An observable for which we generate the predictions - the nuclear modification factor $R_{A A}$ is defined [15] as the ratio of the quenched $A+A$ spectrum to the $p+p$ spectrum, scaled by the number of binary collisions $N_{b i n}$ :

$$
R_{A A}\left(p_{\perp}\right)=\frac{d N_{A A} / d p_{\perp}}{N_{b i n} d N_{p p} / d p_{\perp}} .
$$

In order to obtain a bare gluon quenched spectra, we used the generic $\mathrm{PQCD}$ convolution (Equation (1) from [16]), where the initial gluon distribution is computed according to [17,18], while radiative energy loss probability includes the multi-gluon [19] and the path-length $[15,16]$ fluctuations.

\section{Results}

In this section we provide analytical and numerical results bsg approximation [11] and also make a comparison with the $s g$ case [12]. After rather involved calculations (for detailed derivation see [11]) we obtained the expression for the bsg single gluon radiation spectrum to the 1st order in opacity:

$$
\begin{aligned}
\frac{d N_{g, b s g}^{(1)}}{d x}= & \frac{C_{2}(G) \alpha_{s}}{\pi} \frac{L}{\lambda} \frac{\left(1-x+x^{2}\right)^{2}}{x(1-x)} \int \frac{d^{2} \mathbf{q}_{1}}{\pi} \frac{\mu^{2}}{\left(\mathbf{q}_{1}^{2}+\mu^{2}\right)^{2}} \int d \mathbf{k}^{2} \\
& \times\left\{\frac{\left(\mathbf{k}-\mathbf{q}_{1}\right)^{2}+\chi}{\left(\frac{4 x(1-x) E}{L}\right)^{2}+\left(\left(\mathbf{k}-\mathbf{q}_{1}\right)^{2}+\chi\right)^{2}}\left(2 \frac{\left(\mathbf{k}-\mathbf{q}_{1}\right)^{2}}{\left(\mathbf{k}-\mathbf{q}_{1}\right)^{2}+\chi}-\frac{\mathbf{k} \cdot\left(\mathbf{k}-\mathbf{q}_{1}\right)}{\mathbf{k}^{2}+\chi}-\frac{\left(\mathbf{k}-\mathbf{q}_{1}\right) \cdot\left(\mathbf{k}-x \mathbf{q}_{1}\right)}{\left(\mathbf{k}-x \mathbf{q}_{1}\right)^{2}+\chi}\right)\right. \\
& \left.+\frac{\mathbf{k}^{2}+\chi}{\left(\frac{4 x(1-x) E}{L}\right)^{2}+\left(\mathbf{k}^{2}+\chi\right)^{2}}\left(\frac{\mathbf{k}^{2}}{\mathbf{k}^{2}+\chi}-\frac{\mathbf{k} \cdot\left(\mathbf{k}-x \mathbf{q}_{1}\right)}{\left(\mathbf{k}-x \mathbf{q}_{1}\right)^{2}+\chi}\right)+\left(\frac{\left(\mathbf{k}-x \mathbf{q}_{1}\right)^{2}}{\left(\left(\mathbf{k}-x \mathbf{q}_{1}\right)^{2}+\chi\right)^{2}}-\frac{\mathbf{k}^{2}}{\left(\mathbf{k}^{2}+\chi\right)^{2}}\right)\right\}
\end{aligned}
$$

where $\alpha_{S}$ denotes a constant strong coupling, $L$ a medium length, $\lambda$ a mean free path, $\chi=m_{g}^{2}(1-x+$ $x^{2}$ ); while $\mathbf{k}$ and $\mathbf{q}_{1}$ stands for a transverse momentum of radiated and exchanged gluons, respectively. To our knowledge, this result presents the first introduction of the effective gluon mass in the $b s g$ approximation radiative energy loss. On the other hand, in the $s g$ case $\frac{d N_{g}^{(1)}}{d x}$ acquired the form [12]:

$$
\begin{aligned}
\frac{d N_{g, s g}^{(1)}}{d x} & =\frac{C_{2}(G) \alpha_{s}}{\pi} \frac{L}{\lambda} \frac{1}{x} \int \frac{d^{2} \mathbf{q}_{1}}{\pi} \frac{\mu^{2}}{\left(\mathbf{q}_{1}^{2}+\mu^{2}\right)^{2}} \int d \mathbf{k}^{2} \frac{\left(\mathbf{k}-\mathbf{q}_{1}\right)^{2}+m_{g}^{2}}{\left(\frac{4 x E}{L}\right)^{2}+\left(\left(\mathbf{k}-\mathbf{q}_{1}\right)^{2}+m_{g}^{2}\right)^{2}} 2\left\{\frac{\left(\mathbf{k}-\mathbf{q}_{1}\right)^{2}}{\left(\mathbf{k}-\mathbf{q}_{1}\right)^{2}+m_{g}^{2}}\right. \\
& \left.-\frac{\mathbf{k} \cdot\left(\mathbf{k}-\mathbf{q}_{1}\right)}{\mathbf{k}^{2}+m_{g}^{2}}\right\} .
\end{aligned}
$$

It can easily be verified that Equation (2): is symmetric under the exchange of two outgoing gluons; and in the sg limit recovers Equation (3). Upon obtaining the analytical expression we addressed the effect of finite $x$ on the numerical predictions. The following set of parameters was used: $\mu=\sqrt{4 \pi \alpha_{s}\left(1+n_{f} / 6\right)} T, n_{f}=3, \alpha_{s}=\frac{g_{s}^{2}}{4 \pi}=0.3, L=5 \mathrm{fm}, \lambda=1 \mathrm{fm}, T=300 \mathrm{MeV}$, to mimic the 
standard LHC conditions. From Figure 1, which corresponds to $\Delta E^{(1)} / E$ and $N_{g}^{(1)}$, we surprisingly observed a small, but of an opposite sign, difference between $b s g$ and $s g$ curves for these variables.
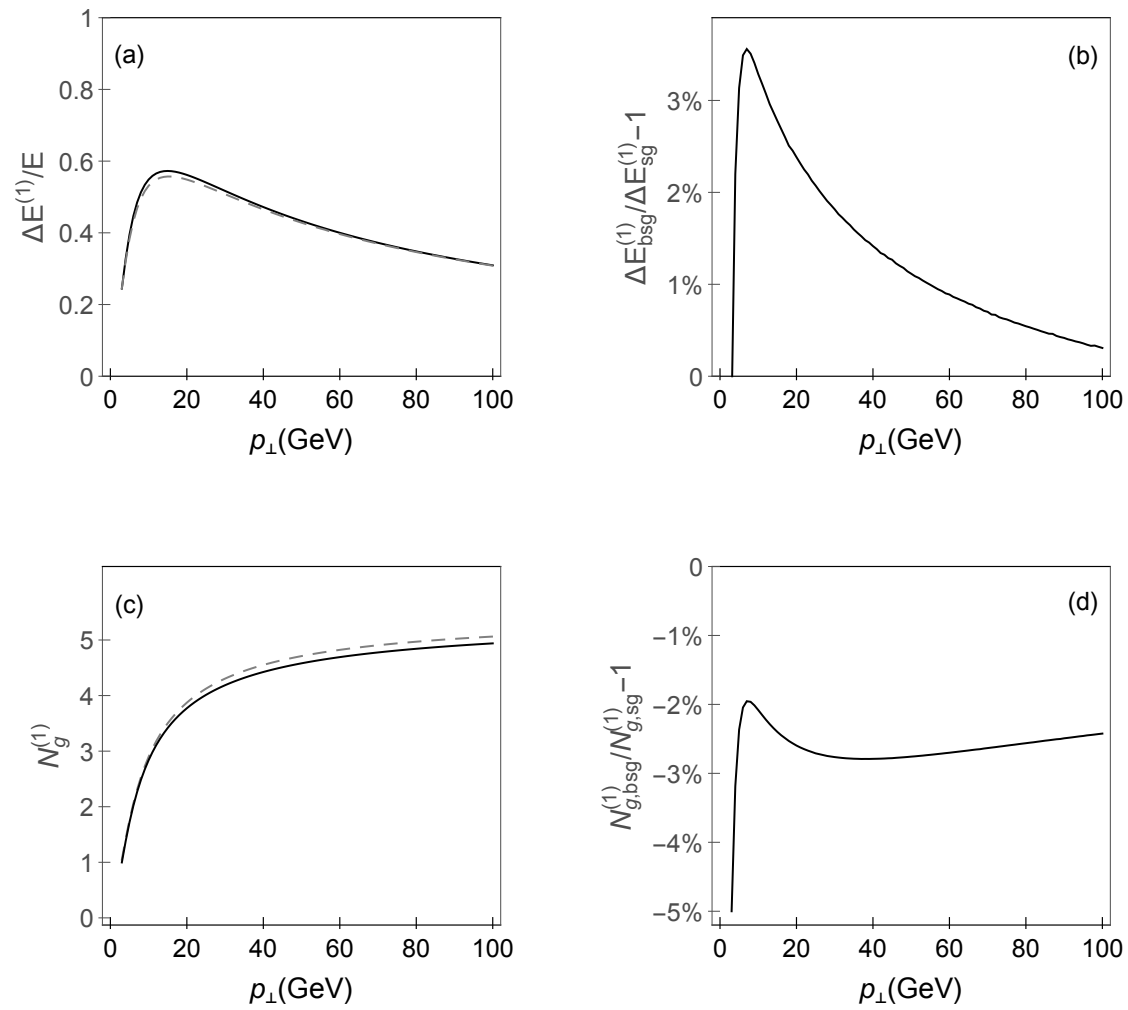

Figure 1. (a) The finite $x$ effect on $\Delta E^{(1)} / E$ and (b) its relative effect on $\Delta E^{(1)} / E$. The same for $N_{g}^{(1)}$ is shown in (c) and (d), respectively. The full (dashed) curve corresponds to the $b s g(s g)$ case. Adapted from [11].

Finally, we addressed how the finite $x$ affects the suppression predictions, by comparing the $b s g$ and the $s g$ gluon $R_{A A}$ predictions in Figure 2 and obtained even smaller discrepancies between these two curves, compared to the previous variables.
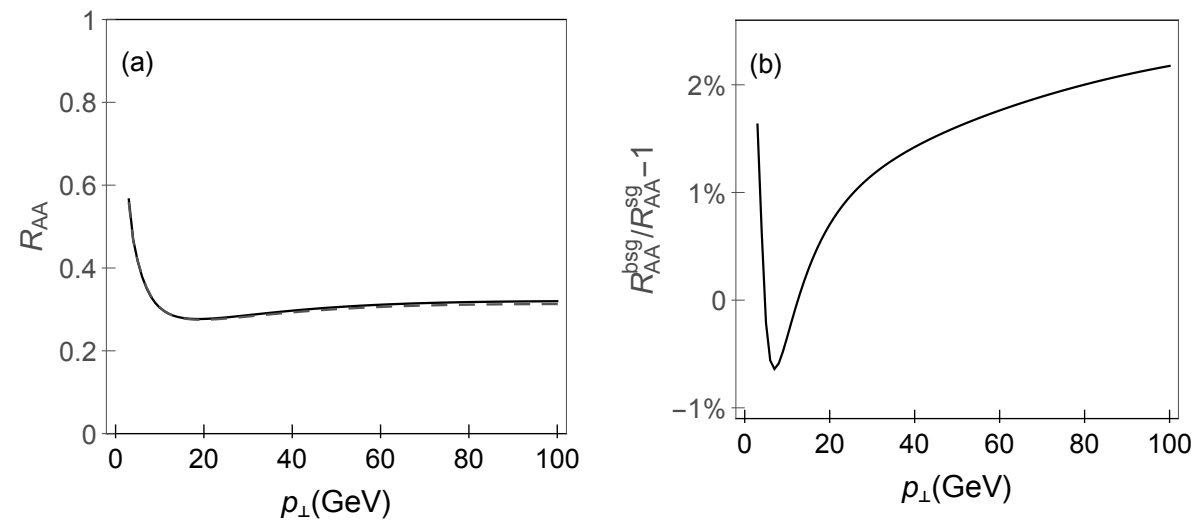

Figure 2. (a) The effect of a finite $x$ on $R_{A A}$ as a function of final $p_{\perp}$. The full (dashed) curve corresponds to the $b s g(s g)$ case. (b) provides a percentage of $R_{A A}$ change when the sga is relaxed. Adapted from [11]. 


\section{Discussion}

Our calculations yielded bsg analytical expression (Equation (2)) which is clearly different, and significantly more complex than in the $s g$ case (Equation (3)). However, we surprisingly obtained small difference for gluon's $\Delta E^{(1)} / E$ and $N_{g}^{(1)}$ numerical predictions in two cases (Figure 1). Furthermore, the effect of finite $x$ is even smaller for $R_{A A}$ (Figure 2). This negligible gluon $R_{A A}$ change could be intuitively explained by the interplay of the opposite effects on $\Delta E^{(1)} / E$ and $N_{g}^{(1)}$, which non-trivially enter $R_{A A}$ (compare the right panels of Figures 1 and 2). Consequently, the high $p_{\perp}$ quarks are even less likely to be affected by this approximation. This implies that within the DGLV formalism the soft-gluon approximation remains well-founded. Finally, we also expect that the sga can be reliably applied when a dynamical medium is considered, which however remains to be rigorously tested in the future.

Funding: This research was funded by the European Research Council, grant ERC-2016-COG: 725741, and by the Ministry of Science and Technological Development of the Republic of Serbia, under project numbers ON171004 and ON173052.

Conflicts of Interest: The authors declare no conflict of interest.

\section{References}

1. Armesto, N.; Salgado, C.A.; Wiedemann, U.A. Medium induced gluon radiation off massive quarks fills the dead cone. Phys. Rev. D 2004, 69, 114003, doi:10.1103/PhysRevD.69.114003.

2. Baier, R.; Dokshitzer, Y.L.; Mueller, A.H.; Peigne, S.; Schiff, D. Radiative energy loss and p(T) broadening of high-energy partons in nuclei. Nucl. Phys. B 1997, 484, 265-282, doi:10.1016/S0550-3213(96)00581-0.

3. Gyulassy, M.; Levai, P.; Vitev, I. Reaction operator approach to nonAbelian energy loss. Nucl. Phys. B 2001, 594, 371-419, doi:10.1016/S0550-3213(00)00652-0.

4. Majumder, A.; Van Leeuwen, M. The Theory and Phenomenology of Perturbative QCD Based Jet Quenching. Prog. Part. Nucl. Phys. A 2011, 66, 41-92, doi:10.1016/j.ppnp.2010.09.001.

5. Apolinario, L.; Armesto, N.; Salgado, C.A. Medium-induced emissions of hard gluons. Phys. Lett. B 2012, 718, 160-168, doi:10.1016/j.physletb.2012.10.040.

6. Zhang, B.W.; Wang, X.N. Multiple parton scattering in nuclei: Beyond helicity amplitude approximation. Nucl. Phys. A 2003, 720, 429-451, doi:10.1016/S0375-9474(03)01003-0.

7. Ovanesyan, G.; Vitev, I. An effective theory for jet propagation in dense QCD matter: jet broadening and medium-induced bremsstrahlung. JHEP 2011, 1106, 080, doi:10.1007/JHEP06(2011)080.

8. Ovanesyan, G.; Vitev, I. Medium-induced parton splitting kernels from Soft Collinear Effective Theory with Glauber gluons. Phys. Lett. B 2012, 706, 371-378, doi:10.1016/j.physletb.2011.11.040.

9. Djordjevic, M.; Djordjevic, M. LHC jet suppression of light and heavy flavor observables. Phys. Lett. B 2014, 734, 286-289, doi:10.1016/j.physletb.2014.05.053.

10. Djordjevic, M.; Djordjevic, M.; Blagojevic, B. RHIC and LHC jet suppression in non-central collisions. Phys. Lett. B 2014, 737, 298-302, doi:10.1016/j.physletb.2014.08.063.

11. Blagojevic, B.; Djordjevic, M.; Djordjevic, M. Calculating hard probe radiative energy loss beyond the soft-gluon approximation: Examining the approximation validity. Phys. Rev. C 2019, 99, 024901, doi:10.1103/PhysRevC.99.024901.

12. Djordjevic, M.; Gyulassy, M. Heavy quark radiative energy loss in QCD matter. Nucl. Phys. A 2004, 733, 265-298, doi:10.1016/j.nuclphysa.2003.12.020.

13. Gyulassy, M.; Wang, X.N. Multiple collisions and induced gluon Bremsstrahlung in QCD. Nucl. Phys. B 1994, 420, 583-614, doi:10.1016/0550-3213(94)90079-5.

14. Djordjevic, M.; Gyulassy, M. The Ter-Mikayelian effect on QCD radiative energy loss. Phys. Rev. C 2003, 68, 034914, doi:10.1103/PhysRevC.68.034914.

15. Dainese, A. Perspectives for the study of charm in-medium quenching at the LHC with ALICE. Eur. Phys. J. C 2004, 33, 495-503, doi:10.1140/epjc/s2004-01645-4.

16. Wicks, S.; Horowitz, W.; Djordjevic, M.; Gyulassy, M. Elastic, inelastic, and path length fluctuations in jet tomography. Nucl. Phys. A 2007, 784, 426-442, doi:10.1016/j.nuclphysa.2006.12.048. 
17. Kang, Z.B.; Vitev, I.; Xing, H. Nuclear modification of high transverse momentum particle production in p+A collisions at RHIC and LHC. Phys. Lett. B 2012, 718, 482-487, doi:10.1016/j.physletb.2012.10.046.

18. Sharma, R.; Vitev, I.; Zhang, B.W. Light-cone wave function approach to open heavy flavor dynamics in QCD matter. Phys. Rev. C 2009, 80, 054902, doi:10.1103/PhysRevC.80.054902.

19. Gyulassy, M.; Levai, P.; Vitev I. Jet tomography of Au+Au reactions including multigluon fluctuations. Phys. Lett. B 2002, 538, 282-288, doi:10.1016/S0370-2693(02)01990-1.

(C) 2019 by the authors. Licensee MDPI, Basel, Switzerland. This article is an open access article distributed under the terms and conditions of the Creative Commons Attribution (CC BY) license (http:// creativecommons.org/licenses/by/4.0/). 